\title{
Über federnde und nicht federnde Subluxation des Ellenkïptchens.
}

\author{
$\operatorname{Vin}$ \\ Protessor Dr. Sonntag. \\ Oherarzt der Polikinik an der chirurgischen Unjersitätsklinit zu Leipzig. \\ Mit 4 Abbildungen im Tert.
}

(Eingegungen am 24. Mai 1921.)

Nachstehender Fall von fodernder Subluxation des Ellenköpfchens, d. h. der [ilna im unteren Radioulnargelenk. dürfte das Interesse der Praktiker verdienen. Der Fall verkürpert ein gut charakterisiertes und wohl nicht außergewöhnliches Krankheitsbild. In den Lehr- und Handbüchern sowie in der Literatur findet sich aber davon wenig oder gar nichts vermerkt. Damit soll nun nicht gesagt sein, daß das Leiden für den Facharzt etwas Unbekanntes bedeutet. Es ist auch bei der Häufigkeit von Verletzungen der Handgelenksgegend anzunehmen, daß das Krankheitsbild, wenn es auch selten ist, dem beschüftigten Praktiker doch hin und wieder begegnet. Letzterer muß3 daher über diese typische Verletzung orientiert sein, zumal sie sich auch ohne Röntgenaufnahme diagnostizieren läßt und der Patient, welchem die charakteristischen Verïnderungen in der Regel auffallen, vom Arzt zu wissen verlangt, um was es sich handelt und was zu tun ist. Über diese Fragen, welche. wie gesagt, in den Lehr- und Handbüchern sich nicht oder nicht genügend beantwortet finden, soll die folgende Besprechung des Leidens an Hand eines selbst beobachteten Falles kurz orientieren.

Krankengeschich te: 19jähriger Landwirt.

Vorgeschichte: Vor einem Jahr Fall auf den linken Handrücken bei pronierter und gebeugter Hand; Arzt stellte Handverstauchung fest und verordnete 14 Tage Ruhe und Lmschlag; Patient arbeitete zugleich weiter. Seit dem Lnfall blieben Schmerzen im linken Handgelenk bein festen Zufassen und besonders beim Schwerheben, z. B. Fuderabstechen.

Untersuchungsbefund: Cnteres Ende der Clna springt auf der linken Seite gegenüber der rechten abnorm vor nach oben (dorsal) und außen (ulnar). Durch direkten Druck mit dem Daumen läßt es sich nach unten (volar) zurückdrücken, und zwar bis in normale Lage; beim Nachlassen des Druckes federt es aber sofort wieder zurück. Linke Handgelenksgegend erscheint im seitlichen sowohl wie im antero-posterioren Durchmesser verbreitert; Umfang in Höhe des Ellenköpfehens ergibt mit dem Bandma:3: rechts 18 $1 \%$ links $19 \mathrm{~cm}$; Messung mit dem Tasterzirkel seitlich (von Radius zu Llna): rechts $5^{1 / 1}$, links $5^{1 / 2} \mathrm{~cm}$ und von oben nach unten: rechts 4 , links $5 \mathrm{~cm}$. Bei Betrachtung von oben 
erscbeint links vor (distal) dem Ulnaköpfchen eine tiefere Furche und der seitliche Umaiß des VInaküpfchens stärker vorspringend als rechts. Im übrigen sind Handgelenk und benachburte Knochen ohne krankhaften Befund. Handbewegungen nach allen Richtumgen fræi. Faustschluß völlig. Händedruck kräftig; Hündedruckkraftmesser rechts und links 34. Hand und Arm linkerseits nicht stärker geschwächt;

\begin{tabular}{|c|c|c|}
\hline Tmfangsmalse & rechts & links \\
\hline Oberarm & $27 \mathrm{~cm}$ & $25_{i=2}^{1} \mathrm{~cm}$ \\
\hline Lnterarn & $27 \quad$ & $26^{1 / 2}$, \\
\hline Sittelhand & $2: 21: 2$, & $231 \%$ \\
\hline
\end{tabular}

Röntgenbild ergibt links eine Verschichung des ¿lnaköpfehens auf dem seit. lichen Bild: nach oben (dorsal) und auf dem Bild von der Fläche: nach auBen (uhar).

Krankhoitsyerlauf: Empfohlen wurden: heiße Wasser- oder Sandbider, Lm. schläge, Pinselungen, Einreibungen, Massige u. dgl, sowie Handgelenksmanschette, bei starken Beschwerdeneventuell Operation (Fixation de's Ellenkijpfchens). Letztere lelunte Patient ab. Cnter der renannten Behandlung und zugleich bei der Arbeit verschwanden die Beschwerden angeblich. In übrigen ergab die Sachuntersuchung nach 7 Wochen denselben Befund.

Uber das im rorgenannten Fall bestehende Krankheitsbild lassen sich in der Literatur, spez. in den Lehrund Handbücherr, folgende Angaben finden: Bei den bekanntlich sehr häufigen Verletzungen der $\mathrm{Handgelenkgegend}$ handelt es sich in der Regel um Kontusionen oder Distorsionen des Hand. gelents oder um Frak-

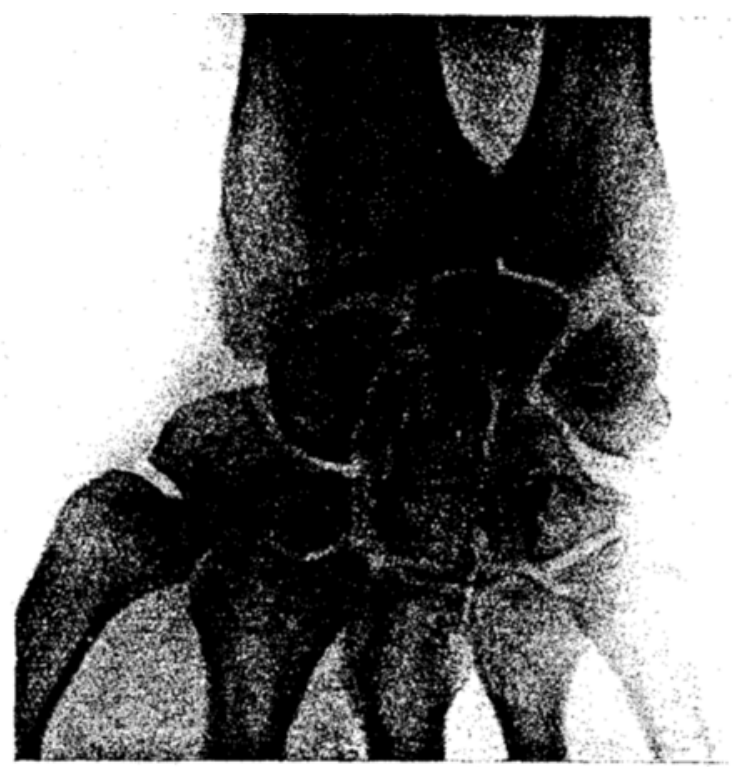

Ab̧. 1. Federnde Luxation des Chaköpfchens. Röntgenbild von der seite und von der Fläche. turen des Radius, verbunden oft mit Fraktur des Griffolfortsatzes der Elle und bisweilen mit Fraktur oder Luxation ron Handwurzelknochen. Isolierte Verletzungen letzterer sind dagegen selten. Sehr selten sind Luxationen des Handgelenks.

Ebenfalls sehr selten sind nach dem übercinstimmenden Lrteil aller Autoren isolierte Luxationender Ulua im unteren Radioulnargelenk. Man muß dabei unterscheiden zwischen vollständigen und unvollständigen Luxationen des Ulnaköpfchens. Von diesen sind die vollständigen Luxationen anscheinend seltener; etwas häufiger dagegen die unvollständigen Luxationen oder Subluxationen des Ulnaköpfchens.

Genauere Angaben über die isolierten Luxationen des UIna. köpfchens finden sich u. a. bei Bardenhener, Tillmanns, Friedrich (Handbuch d. prakt. Chir.). Das Ulnaköpfchen kann sich nach vorn oder nach 
hinten verlagern; es kann auch die Haut durchbohren, in welchem Fall die Gefahr der Handgelenkrereiterung droht und eventudl dic Reselition des Köpti-hens nëtig wircl. Welehes Vorkommmis nicht ganz selten. u. a. auch vor riniger Ztit herr boobachtet ist. Die Entstehung ist selten indirekt rturch Fall anf dir Hand, meist direkt durch Stols gregen das untere Fnde des Vorderams. Anatomischo Cintersuchungen iuber die Verletzung fehlen;

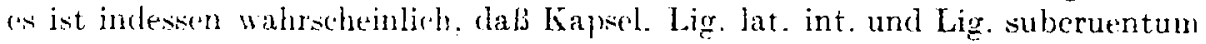
zerrissen sind: jeflenfalls spricht rlie starke Boweglichkeit und die Rezidivneigung dafür. Reposition ist leicht. Petention schwierig: es empfiehlt sich fester Verband bis zu drei IFonaten in Supination oder in permanenter Ex-

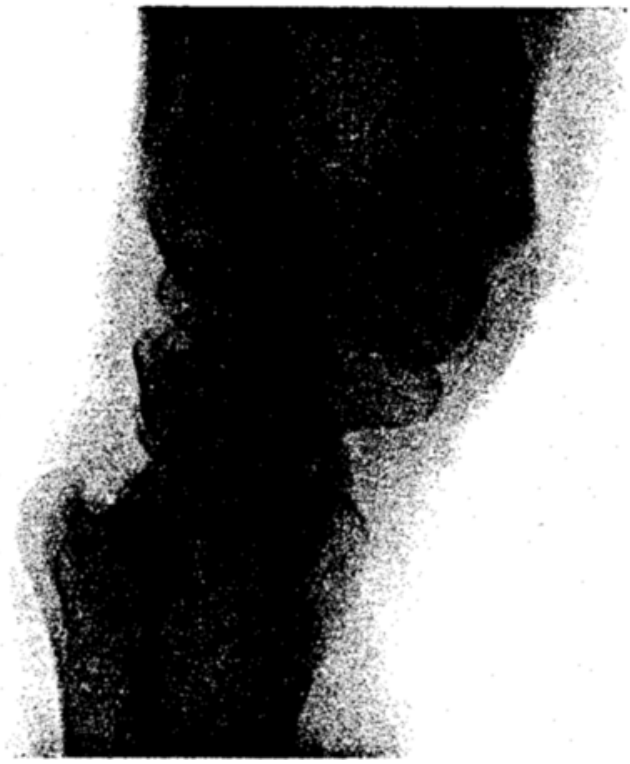

Abh, 2. Federnde Luxation des Chakeipfebens. Räintuenbild ron der sirite und ron der Fläiches. tension, in veralteten Fillen mit bedeutender Funktionsstörung: Roseltion des L'naköpfehens. Isolierte subluxationendes Llnaköpfehens sol. lern nach Goyrand infolge Zerreißung der Cartilago triangularis bei kleinen Kindern rorkommen, welehe an der Hand howhezert werden. Ferner ar. wïrnt $H$ olferich auch ihr Vor. sommen bei Wäscherinuen infolge Wäscheanswindens.

Nicht allzu selten sollen Luxationen und subluxa. tionen des UInaköpfohens bri dem typischen Radiusbruch sein (Bardenheuer u. a.). Thon (Deutsche Zeitschr f. Chir. 81) erwähnt darüber .. Bei Fillen ron typischen Riadiusfrakturen, bej welchen die Bruchlinie so verlauft. daß die Gelenlifliche für die Llna noch in ren Bereich der Fraktur fällt. erfolert res öfteren eine schwere Versehiehung im sinne einer Luxationsstellung der Llna: rerläutt dagegen die Bruchlinie bei der typischen Radiustraktur derart, dals rie Unagelenkflithe am Radius intakt bleibt, so sind auch Luxationen der Llna sehr selten." Besonders bei dem (allerdings ziemlich seltenen) Bruch der ulnaren Kante des Radius ist naturgemäß eine Verschiebung des Clnaköpfchens leicht möglich; als derartiger Fall kann auch der unten wiedergegebene gelten. Lilienfeld (Arch. f. kin. Chir. 82, 1907) empfiehlt bei dem klassischen Radiusbruch auf das Rarlioulnargelenk immer die Aufmerksamkeit zu richten, da eine bisher wenig gewürdigte, aber nicht seltene Komplikation die Lostrennung und Verschiebung des Clnaköpfchens sti. Er fand sie unter 115 Radiusbrüchen binal in so ausgesprochener Weise. daß man sie fast als Luxation der Clna bezeichnen könnte, und bringt ein diesbrzägliches Röntgenbila. An Hand eines weiteren Röntgenbildes fordert 
er besondere Beachtung der Clnaverschiebung bei der Repmition wogen der Gefahr sehr schwerwiegencier Nachteile infolge exzessiven Wachstums der LIna bei Jugendlichen. Daß bei gleichzeitiger Radiusfralitur das Clnakijptchen hisweilen luxiert, aber wohl hä̈utiger nur subhuxiert unter ZerreiBung des Discus articularis und des Lig. subrrentum, erwähnt anch $\mathrm{Hoffat}$. Leter u. a. Tst dabei die Clna unter Ruptur des Discus articularis betrichtlich nach auBen abgewichen, so gelingt, wie Fricdrich betont, ab und zu dit Forrelitur der dadurch entstandenen Deformitiit ïberhaupt nur unvolletändig.

Nach Tillmanns findet gleiches: auch bei schweren Handrerstauchungen bisweilen statt. Bardenherer sagt darüber: Ohnckomplikation mit Radiusfraktursind Lidationen des binaköpfchens außerordentlich selten: sie bleiben meist inkomplett. Stern (Beitr. zur klin. Chir. 117, 1919) bemerlit, daB bei forcierter Volarflexion der Hand es leicht zur Zerreißung des Lig. collaterale ulnae und der Cartilago triangularis kommt und hierbei häufig eine Subluxation, seltener eine Luxation auftritt, und zwar nach dorsal, entsprechend der Versehiebung des zentralan Fragments des Radius; er bringt einen entsprechendern Fall mit Röntgenskizzc. sowie je einen Fall irolierter Dorsalluxation ron Nove und ron $H$ offa: auber durch forcierte Volarflexion könne die Dorsal. luxation auch auftreten durch forcierte Pronation ofler durch Supination der Hand bei fixiertem Lnterarm und Pronation des Cnterarms bei fixierter Hand. Vert. zitiert die Leichenversuche ron Hönigsehmidt (Deutsche Zeitschr. t. (hir. 10, 1878) bzw. Bonnet (Gevenk-

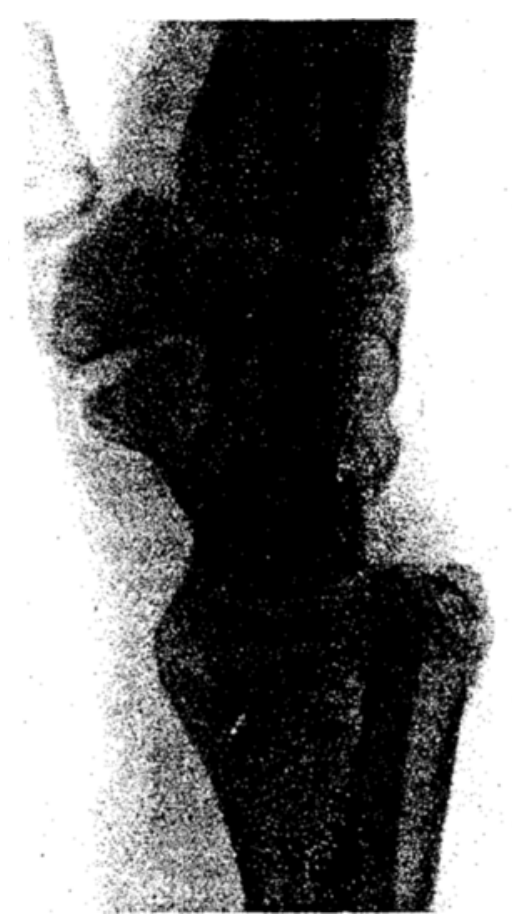

Abb. 3. Subluxationsstellung des Ellenköjfehems nach oben nebst fraktur dey ulnaren kante des Radius. Räntgenbila ron eler seite. krankheiten 1847). Danach kommt durch forcierte Rardialabduktion. Wenn auch beine luxation, aber roch ein stirkeres Vorspringen und eine abnorme Dislozierbarkeit des muteren CInaendes vor infolge Risses des Lig, coliat. uhao oder des Dreieckluorpels an seiner Insertion am Radius. Aus Vorgenanntem ergibt sich. dals auch ohno Rarliusbrueh bei sog. Handverstauchung infolge Risses des Drejeckknorpels bzw. seines Bandes oder des tinaseitenbandes sowohl Luxationen wie subluxationen vorkommen können. Fälle ron sublixation, und zwar je einer dorsal und uluar. sind weiter unten wiedergegeben worden, wovon der erstere auch einen kleinen Abbruch an de:r ulnaren Kante des Padius aufwcist und oben schon genannt ist.

Sonst findet sich in der Literatur über die Luxation und Subluxation, insbesondere auch äber die federnde Subluxation dis Ellenköpf- 
'hens. nichts Weiteres erwähnt. Lnter diesen Lmständen erscheint der oben besehriebene Fall von federnder Subluxation des Ellenköpfehens einer genaueren Mitteilung unter Wiedergabe des Pöntgenbildes für den Praktiker wert.

Gleichzeitig wurde je ein Fall ron Subluxationsstellung des Ellenköpfchens nach oben (dorsal) und ein solcher nach auBen (ulnar) beobachtet, aber beide ohne das Symptom des Federns. Die Diagnose der subluxation ist hit'r auch im Röntgenbild zu stellen, und zwar im Falle dorsaler Subluxation auf dem scitlichen Bild (das Bill ron der Fläche läbt grleichzeitig eine geringe Knochenverletzung de's Radius am Radionlnargelent: an der sog. ulnaren Kante des Radius erkennen) und im Falle ulna ror Subluxation auf dem Bild ron der Fläche (gleichzeitig "rocheint eine bleine

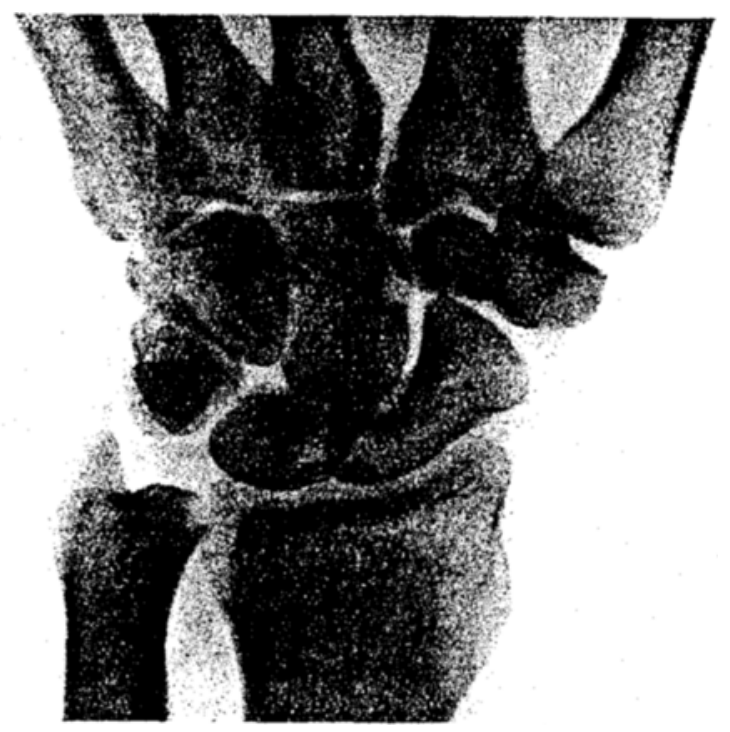

Abb. 4. Subluxationsstellung des Ellenkijpfehens nach auBen. Röntgenbild ron der Flaiche.

Aussparung an der radialen Kante des Ellenköpfchens). Im übrigen, d. h. klinisch, erogibt sich die Diagnose aus der sichtharen Abweichung des Ellenköpfchens nebit entsprechender Vermehrung des Unterarnumfanges. Ueist wirl aber wohl nur die gleichzeitig bestehende Handverstauchung onter Radiusfraktur beachtet. Fälle solcher Art sind wohl nicht allzu selten, sei es mit. sei es ohne Radiusbruch. Den Patienten dürfte - wir in unseren Fällen das Vorstehen des Ellenköpfehens selbst auffallen, und zwar einige Zeit nach der Verletzung. nachdem die zunächst bestehende Schwellung zurückgegangen ist. Anscheinend bestehen fortdauernde Beschwerden, welche hartnäckiger sind alis bei den meisten Fiillen ron Handverstauchung oder Speichenbruch. Site sind wohl zum Teil auch bedingt durch Komplikationen: Kapsel-, Band- und Sehnenverletzungen.

Für das Kranliheitsbild der federnden Subluxation des Ulnaköpfchens ergeben sich in Kürze folgende praktisch wichtigen Leitsätze: 
Vorkommen der Verletzung ist anscheinend selten. Symptomatik und Diagnose ergibt sich aus der typischen Abweichung des Ulnaköpfehens im Verein mit dem Fellern desselben: Röntgenbild sichert die Diagnose und kliirt auch über gleichzeitige Knochenverletzungen auf. Therapeutisch genïgt wohl meist symptomatische Behandlung nebst fester (Leder-)Bandage ums Handgelenk: erwägenswert, aber wohl recht kompliziert erscheint eine Bandage, wie sie Mayer (Ärztl. Sachrerständigen-Ztg. 1896, 22) bei einer rezidivierenden Luxation im unteren Radioulnargelenk anwandte: stark federndes, imnen gepolstertes Armband aus Stahl mit Öfnung für die Radialseite und mit auf- und abschiebbarer Pelotte für clas Clnaköpfchen. Bei fortdauernden und starken Beschwerden kommt u. U. Operation in Frage: Fixation des [Inaköpfchens durch Bandnaht oder sicherer durch Knochennaht eventuell mit Faszienverstälsung, ausnahmsweise (z. P. bei infizierter Wunde) Resektion. Auf die $\mathrm{Pathogenese} \mathrm{soll} \mathrm{nicht} \mathrm{eingegangen} \mathrm{werden;} \mathrm{Voraussetzung}$ des Ieidens ist Verletzung der entsprechenden Bandverbindungen des Ulnakïpfehens, ron demen in Frage kommen: Capsula artic. radio-ulnaris distalis (schlaff!), Diseus artic. s. Cartilago triang. (zwischen Incisura uln. radii und Ulna), Lig. interosseum (?), Lig. triang. s. subcruentum (zwischen Band. scheibe und Proc. styl. ulnae), Lig. lat. int. s. collat carpi uln. (zwischen Proe. styl. ulnae und Os triquetrum). Ligg. ulnocarp. vol. a. dorsale und Lig. carpi dorsale. Daß nach Lockerung hzw. Zerreißung seiner Bandverbindungen. z. B. des Clnaseitenbandes, das Clnaköpfchen subluxiert. erklït sich durch seine große Beweglichkeit infolge Laxheit seiner Bandrerbindungen bei Fehlen eines unmittelbaren Kontakts mit der Handwurzel und bei kleiner Gelenkfläche zum Radius: die Richtung der subluxation nach oben (rlorsal) oder nach außen (ulnar) oder nach beiden Gegenden zugleich ergibt sich aus der Tatsache. daß das Clnaköpfohen dahin mangels Bederkung durch teste Weichteile leicht ausweichen kann. Nach welcher Richtung die Subluxation erfolgt und ob sie federt oder nicht, hängt im übrigen davon ab, welche Bandverbindungen und in welnhem Iaße sie zerrissen sind.

In Analogie ist die federnde Subluxation res Fllembëpfchers zu setzen u. a. mit der des akromialen Endes des Schlüsselbeins. 